\title{
Electromagnetic Properties of Melt-Grown YBaCuO Superconductors Doped by Gd and Sm
}

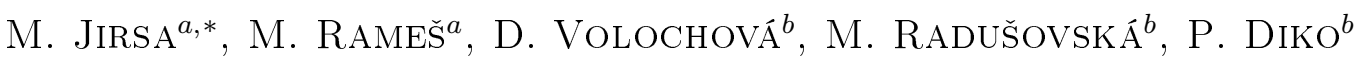 \\ ${ }^{a}$ Institute of Physics, ASCR, Na Slovance 2, CZ-182 21 Praha 8, Czech Republic \\ ${ }^{b}$ Institute of Experimental Physics SAS, Watsonova 47, 04001 Košice, Slovakia
}

\begin{abstract}
Electromagnetic properties of $\mathrm{YBa}_{2} \mathrm{Cu}_{3} \mathrm{O}_{y}$ samples melt-grown in air and doped with small amounts of light rare earth (LRE) ions Gd and Sm were studied. The LRE doping creates a point-like disorder contributing to the second peak on the magnetization curve. In the field range of the second peak the average magnetic moment, $M_{a v}$, deduced from the magnetization curves exhibited strong fluctuations. $M_{a v}$ is commonly regarded as an equilibrium moment close to the thermodynamic reversible one, obeying in intermediate fields a logarithmic field dependence. However, in our experiments $M_{a v}(B)$ curves failed to follow such a dependence below irreversibility line. $M_{a v}(B)$ curves deduced from rather static measurements, done by SQUID magnetometer still showed the disturbance but significantly weaker. It indicates that this feature reflects the vortex dynamics in the second peak region. Its scaling with temperature was found to coincide with that of the pinning force.
\end{abstract}

DOI: 10.12693/APhysPolA.126.326

PACS: 74.25.Ha, 74.25.Op, 74.25.Sv, 74.60.Ge, 74.72.-h

\section{Introduction}

In attempt to search for new vortex pinning media and structures, we studied the effect of doping of $\mathrm{YBa}_{2} \mathrm{Cu}_{3} \mathrm{O}_{y}$ by small amounts of rare earth ions Sm and Gd. In the present paper we report on the critical current density and the average magnetic moment calculated from magnetic hysteresis loops of the samples.

\section{Experimental details}

Single-grain pellets of $\mathrm{YBa}_{2} \mathrm{Cu}_{3} \mathrm{O}_{y}, \mathrm{YBa}_{2} \mathrm{Cu}_{3} \mathrm{O}_{y}+$ $1 \mathrm{~mol} \% \mathrm{Sm}$, and $\mathrm{YBa}_{2} \mathrm{Cu}_{3} \mathrm{O}_{y}+1 \mathrm{~mol} \% \mathrm{Gd}$ with a diameter of $2 \mathrm{~cm}$ and $1 \mathrm{~cm}$ thick were prepared by melt growth texturing in air, from the oxides mixed in stoichiometric ratios, with a slight excess of $\mathrm{Ba}$. From the pellets annealed at $400{ }^{\circ} \mathrm{C}$ in flowing oxygen for one week, small samples for magnetic measurements, with dimensions of $1.5 \times 1.5 \times 0.5 \mathrm{~mm}^{3}$, were cut. The experimental data were measured by vibrating sample magnetometer (VSM) in Physical Property Measuring System (9 Tesla magnet) and SQUID magnetometer with 7 Tesla magnet. The critical temperature, ranging between $91 \mathrm{~K}$ and $92 \mathrm{~K}$, was identified by SQUID. All measurements were performed in magnetic field aligned parallel to $c$-axis.

\section{Experimental data}

The critical current densities deduced from magnetic hysteresis loops measured between 90 and $70 \mathrm{~K}$ are shown in Fig. 1; (a) $\mathrm{YBa}_{2} \mathrm{Cu}_{3} \mathrm{O}_{y}$, (b) $\mathrm{YBa}_{2} \mathrm{Cu}_{3} \mathrm{O}_{y}+1 \mathrm{~mol} \%$ $\mathrm{Sm}$, and (c) $\mathrm{YBa}_{2} \mathrm{Cu}_{3} \mathrm{O}_{y}+1 \mathrm{~mol} \% \mathrm{Gd}$. The presented results indicate that the doping by both LRE ions leads to a critical current density enhancement in the whole irreversibility range. On the other hand, the doping causes a decrease of irreversibility field and the second peak position. With $\mathrm{Gd}$, both these effects are stronger. It is

\footnotetext{
*corresponding author; e-mail: jirsa@fzu.cz
}

still a question if this behaviour has something common with the value of paramagnetic moment per free LRE ion, which for $\mathrm{Sm}$ is $0.845 \mathrm{Bohr}$ magnetons $\left(\mu_{\mathrm{B}}\right)$ and for Gd $7.947 \mu_{\mathrm{B}}$, or whether this correlation is only casual.
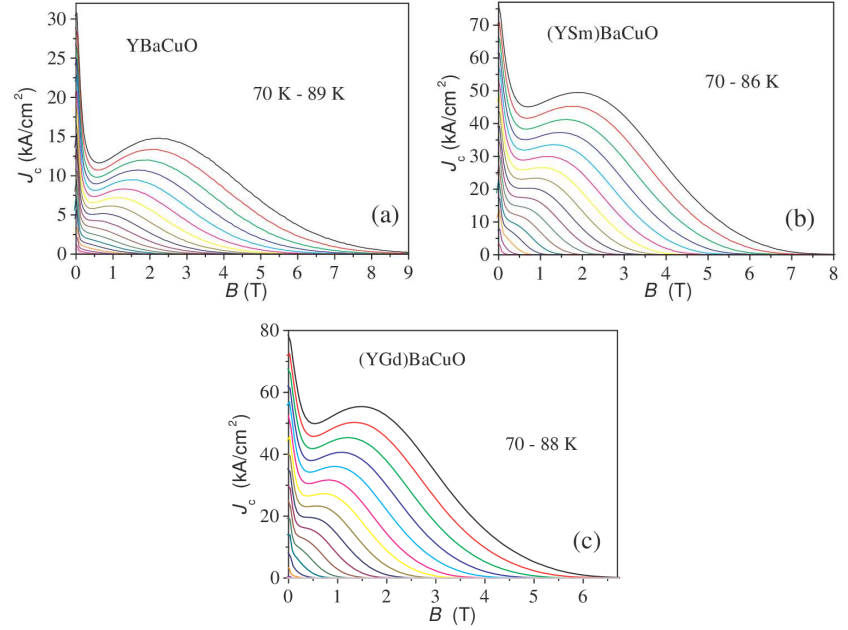

Fig. 1. Critical current densities of $\mathrm{YBaCuO}$ (a), $\mathrm{YBaCuO}+1 \mathrm{~mol} \% \mathrm{Sm}$ (b), and $\mathrm{YBaCuO}+1 \mathrm{~mol} \%$ Gd (c) measured between $70 \mathrm{~K}$ and $T_{c}$. (the highest indicated $T$ ).

Further, we calculated the average magnetic moment, $M_{a v}$, of the upper and lower branches of magnetic hysteresis loops. This moment is usually considered equivalent to the thermodynamic reversible magnetic moment. In the present experiments all three samples exhibited strong fluctuations of the $M_{a v}(B)$ dependence below irreversibility field, $B_{i r r}$, (Figs. 2 and 3). The fluctuations grew with decreasing temperature and developed a peak, which shifted to higher fields, together with increasing $B_{i r r}$ (Fig. 1). We found that the fluctuation $M_{a v}(B)$ 


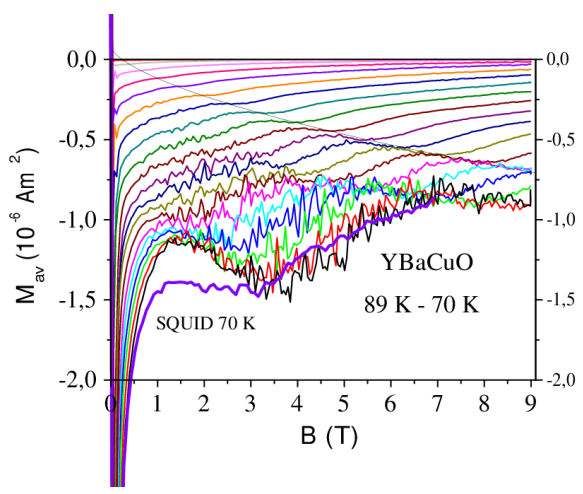

Fig. 2. The $M_{a v}(B)$ curves for $\mathrm{YBaCuO}$ sample at the temperatures given in the panel (VSM data). Below the $B_{i r r}$ line, indicated by the full curve crossing the $M_{a v}(B)$ curves, the average moment exhibits a strong disturbance. For comparison, the full thick curve indicates much less disturbed SQUID data at $70 \mathrm{~K}$.

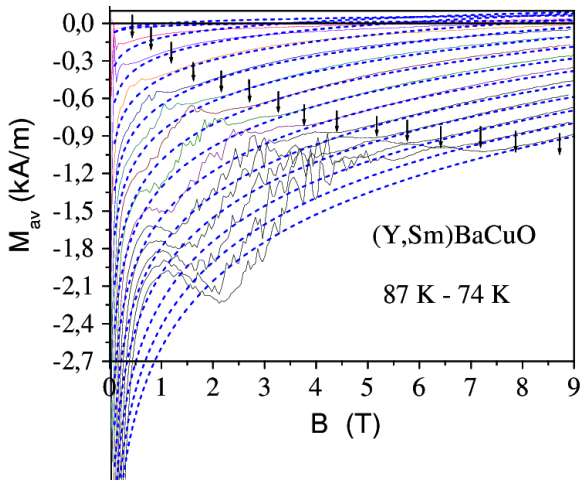

Fig. 3. Fits (the dotted curves) of the $M_{a v}(B)$ curves for $(\mathrm{Y}, \mathrm{Sm}) \mathrm{BaCuO}$ above the irreversibility field (indicated by arrows).

peak scaled with temperature in the same manner as the pinning force associated with the MHL. We also made a parallel experiment by SQUID magnetometer (the thick solid curve in Fig. 2). There, the $M_{a v}$ disturbance was much weaker, which probably reflected the much more relaxed magnetic state of the sample than in the VSM experiments.

Doping by the light rare earth ions introduces a paramagnetic background into the magnetic data. Especially at high magnetic fields and low temperatures the paramagnetic moment might be comparable or even higher than $M_{a v}$. While the paramagnetic background falls out from the critical current density analyses (associated with the irreversible magnetic moment), it stays a part of the $M_{a v}(B)$ data. The Sm and Gd doping brought about $10 \times$ and $100 \times$ higher paramagnetic backgrounds, respectively, than the diamagnetic one (in absolute values), observed in the pristine $\mathrm{YBaCuO}$ (subtracted before plotting the data in Fig. 2).

The fluctuations in $M_{a v}(B)$ below $B_{i r r}$ hindered the use of $M_{a v}(B)$ for determination of thermodynamic parameters in this field range. Only the parts above $B_{i r r}$ could be explored. There, the $M_{a v}(B)$ curves, corrected for the paramagnetic background extrapolated from temperatures above $T_{c}$ and re-calculated for magnetization in $\mathrm{kA} / \mathrm{m}$ units, were fitted by the logarithmic dependence $k_{1} \ln \left(B / k_{2}\right)$ derived for intermediate magnetic fields [1] (Fig. 3). From the fitting parameters $k_{1}$ and $k_{2}$ the thermodynamic quantities $B_{c 1}=2.6 \mu_{0} k_{1} \ln \kappa$ [T], $B_{c 2}=$ $k_{2} / 1.44[\mathrm{~T}], \xi=21.77 / \sqrt{ } k_{2}[\mathrm{~nm}], \kappa=326.16 \sqrt{ }\left(k_{2} / k_{1}\right)$, and $\lambda=7.1 \times 10^{3} / \sqrt{ } k_{1}[\mathrm{~nm}]$ were deduced [2,3]. Both $B_{c 1}(T)$ and $B_{c 2}(T)$ data should obey $\left(1-\left(T / T_{c}\right)^{2}\right)$ dependence close to $T_{c}$. It is the case for all three samples (Fig. 4). The zero temperature coherence length $\xi_{0}$ obtained from the relation $\xi(T)=\xi_{0} / \sqrt{ }\left(1-\left(T / T_{c}\right)^{2}\right)$ reached in $\mathrm{YBaCuO} 1.92 \mathrm{~nm}$, in accord with $(\mathrm{Nd}, \mathrm{Eu}$, Gd)-123 samples [3, 4]. In contrast, in both LRE-doped samples $\xi_{0}$ was about $2.2 \mathrm{~nm}$. In the $\mathrm{YBaCuO}$ sample $\kappa$ reached 98 , close to 100 , considered generally for cuprate superconductors; in both LRE-doped samples, the $\kappa$ value was only about 65 .

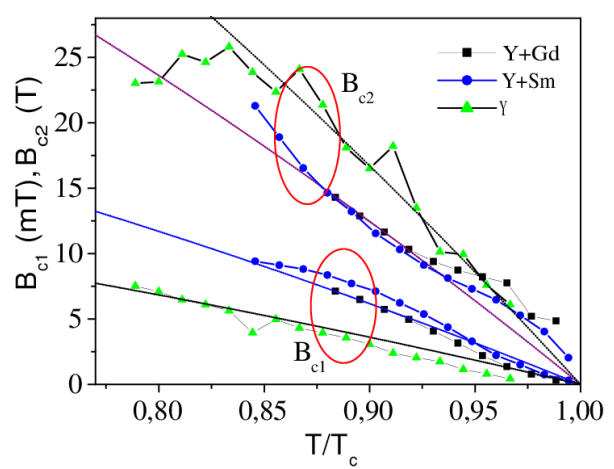

Fig. 4. Critical fields $B_{c 1}$ and $B_{c 2}$ as a function of temperature in vicinity of $T_{c}$. Dotted lines display the adequate $\left[1-\left(T / T_{c}\right)^{2}\right]$ dependences.

\section{Conclusions}

The Sm and Gd doping did not practically affect $T_{c}$. However, both ions caused a significantly enhanced critical current density accompanied with a reduction of $B_{i r r}$. Both elements also caused nearly the same decrease of $\kappa$. This effect was associated with nearly $10 \%$ increase of $\xi_{0}$ and decrease of $\lambda_{0}$ with respect to the pristine $\mathrm{YBaCuO}$ sample.

\section{Acknowledgments}

This work was supported by the project P107/11/0391 of GA, Czech Republic, and the project VEGA 02/0090/13.

\section{References}

[1] Z. Hao, J.R. Clem, Phys. Rev. Lett. 67, 2371 (1991).

[2] M. Jirsa, M. Rameš, M. Muralidhar, D. Volochová, P. Diko, Physics Procedia 45, 65 (2013).

[3] M. Jirsa, M. Rameš, Th. Wolf, J. Phys: Conf. Series 400, 022039 (2012).

[4] M. Jirsa, M. Rameš, Ch. Marcenat, Th. Wolf, J. Supercond. Novel Magn. 26, 901 (2013). 